\title{
Utilización de aplicaciones en dispositivos móviles para el estudio de la geografía turística y la cultura y patrimonio
}

\author{
Laura Victoria Parra Perilla ${ }^{12}$ \\ Raúl Francisco Mateus Tovar ${ }^{13}$ \\ Nancy Patricia Caviedes ${ }^{14}$
}

\section{Introducción}

$\mathrm{L}$ a implementación de diferentes estrategias educativas ha suscitado diversos análisis y movimientos que conllevan a la motivación de participación de los estudiantes en el aula; la educación ha ido evolucionando con el paso de los años, distintos modelos han surgido de los procesos de cognición y la manera en que sus actores se relacionan entre sí. Si bien el estudio de la educación hace parte de las ciencias humanísticas, su aplicación permea todas las áreas e involucra el relacionamiento y los procesos derivados entre el estudiante y el docente, teniendo como objetivo principal promover el aprendizaje y el desarrollo humano a partir de diferentes métodos y herramientas que se utilicen para este fin. La tecnología permite que estos nuevos procesos sean más dinámicos y mejoren el proceso de obtención del conocimiento.

Los dispositivos móviles, como los teléfonos celulares, han transformado en los últimos años la cotidianidad de los estudiantes, y estos no son ajenos a esta realidad; en su mayoría utilizan teléfonos inteligentes, no solo como herramienta de comunicación, sino que este tipo de tecnología también permite a los usuarios disfrutar de un alto volumen de contenido multimedia y generar, a su vez, interacción entre dispositivos. Este tipo de interacciones pueden ser aprovechados dentro del aula de clase por los profesores como instrumento de aprendizaje con los estudiantes en diversas temáticas y, a su vez, sirve para

12. Docente de la Institución Universitaria Politécnico Grancolombiano, Facultad de Ciencias Administrativas, Económicas y Contables, Departamento Académico de Turismo, Grupo de Investigación:" Economía, Derechos y Globalización" Bogotá-Colombia 2018. correo electrónico: HYPERLINK "mailto:Iparrape@poligran.edu.co" lparrape@poligran.edu.co

13. Docente de la Institución Universitaria Politécnico Grancolombiano, Facultad de Ciencias Administrativas, Económicas y Contables, Departamento Académico de Turismo, Grupo de Investigación:" Economía, Derechos y Globalización" Bogotá-Colombia 2018. correo electrónico: HYPERLINK "mailto:rfmateust@poligran.edu.co" rfmateust@poligran.edu.co

14. Docente de la Institución Universitaria Politécnico Grancolombiano, Facultad de Ciencias Administrativas, Económicas y Contables, Departamento Académico de Turismo, Grupo de Investigación:" Economía, Derechos y Globalización" Bogotá-Colombia 2018. correo electrónico: HYPERLINK "mailto:caviedec@poligran.edu.co" caviedec@poligran.edu.co 
encontrar herramientas que sean aprovechadas para adquirir conocimiento. De esta forma, los dispositivos móviles no son solo distractores en el aula de clase, sino que pueden ser dinamizadores en el proceso de enseñanza aprendizaje.

La innovación educativa no pasa simplemente por implementar herramientas aplicadas a la clase o por la inclusión de TIC en el proceso de enseñanza y aprendizaje; se basa también en la posibilidad de renovar métodos para que los estudiantes obtengan fácilmente el conocimiento por medio de procesos armonizados en los que es protagonista, y utiliza diversos mecanismos para la construcción del conocimiento. Paralelamente, en este nuevo paradigma el docente convierte su rol en el de mediador entre el conocimiento y el estudiante, y por tanto entrega recursos, resuelve dudas y fundamenta su acción en los procesos cognitivos del alumno.

El aprovechamiento de las herramientas tecnológicas se implementó con los estudiantes de las asignaturas Geografía turística y Pasajes II de los programas que pertenecen a la Escuela de Gestión del Politécnico Grancolombiano; el objetivo fue permitir a los estudiantes interactuar con aplicaciones tipo juego, permitiendo desarrollar destrezas y fortalezas en conocimientos de geografía y cultura general.

En el presente capítulo se analizará las diferentes aplicaciones/juegos seleccionados para hacer uso de los dispositivos móviles, así como los resultados obtenidos por los estudiantes en las pruebas de aplicación de los conocimientos adquiridos con la herramienta Socrative. Una vez presentados los hallazgos, se busca identificar el resultado del aprendizaje.

\section{Incorporación de tecnologías móviles en el aula de clase}

El uso de la tecnología digital en las aulas es cada vez más apremiante, debido a las características de los jóvenes, personas que nacieron con el boom informático. No obstante, estas transformaciones traen un choque generacional, que se traduce en la resistencia de sectores más conservadores que mantienen la tradición en el aula. De ahí que en este trabajo se plantee que los cambios sean progresivos, y que el docente siga desarrollando su importante tarea al frente de la educación e incorpore las nuevas tecnologías de forma escalonada. El uso de las tecnologías de la información para los docentes no es fácil, y más cuando se tiene como imaginario que pueden ser distractores dentro del aula de clase, ya que le pueden permitir al estudiante perder la atención de la materia que se está desarrollando.

La necesidad que motivó el ejercicio que se presenta a continuación es que los estudiantes objetivo de la metodología pertenecen a los programas de Tecnología en Gestión de Servicios para Aerolíneas, y que una de las competencias fundamentales que requiere este grupo tiene que ver con desarrollar habilidades para el correcto uso de la geografía turística 
en lo relativo a la aplicación de reservas, creación de itinerarios y la asesoría de viajes.

La Geografía turística hace parte del programa y se encuentra parametrizada como una materia común de la Facultad de Negocios, Gestión y Sostenibilidad, tiene 3 créditos educativos y es una materia de primer semestre. En el currículo se concibe como un espacio que permite reforzar los conocimientos y las competencias de las temáticas de la materia Pasajes II.

La geografía nos permite estudiar paisajes y territorios integrando y vislumbrando factores naturales que son integrados a partir de características propias y particulares de un espacio, y donde interactúan con elementos que con efecto del accionar de un grupo de humanos puede terminar siendo objeto de estudio (Alonso, 2003).

La geografía turística o geografía del turismo es el estudio derivado de la ciencia de la Geografía, que permite analizar la práctica turística a partir del reconocimiento de un espacio turístico, analizando el entorno y los factores que lo rodean, como son los atractivos propios de un territorio, destino región o lugar donde el turista ejerza y realice actividades propias del turismo, como por ejemplo una caminata por una montaña, una práctica de torrentismo en una cascada, realizar buceo en el mar, entre otros (Boullon, 2014).

Por lo anterior, surge la necesidad de que los estudiantes aprendan de una manera dinámica todos los elementos que caracterizan el estudio de la geografía turística, tales como:

- Ubicación geográfica: permite al estudiante la construcción de itinerarios mediante la ubicación espacial y la organización lógica de los recorridos de los viajeros.

- Países, capitales: permite al estudiante conocer los diferentes países del mundo y reconocer las capitales, los centros de movilidad a destinos y principales recursos turísticos del mundo.

- Códigos IATA (ISO): son utilizados para identificar de una forma abreviada los principales países, ciudades y aeropuertos; se usan dentro del lenguaje aeronáutico y están implementados dentro de los GDS, que son sistemas de reservas manejados por aerolíneas y agencias de viajes.

- Banderas: permite al estudiante identificar las banderas que representan los diferentes países, que son utilizadas en temas de visado y en reconocimiento de banderas de aeronaves.

- Lugares turísticos: permite al estudiante identificar y ubicar espacialmente los diferentes destinos turísticos en el mundo.

\section{Descripción de la metodología}

Dentro de las aulas de Geografía turística y Pasajes II se desarrolló la implementación de la estrategia metodológica. Se encontró que el celular es un recurso que podría ser incluido 
dentro del desarrollo de las temáticas relacionadas con la asignatura, sin embargo, se necesita realizar un censo inicial para determinar cuál es el nivel de utilización de los teléfonos celulares y con qué tecnologías estaban operando, ya que la identificación del perfil de la población de estudiantes que utilizan el dispositivo permitirá elaborar las estrategias que se usarán dentro de la metodología que se estructura en tres pasos: un ejercicio de evaluación diagnóstica, la aplicación y la evaluación final (ver ilustración 1).

Ilustración 1. Esquema general del planteamiento metodológico

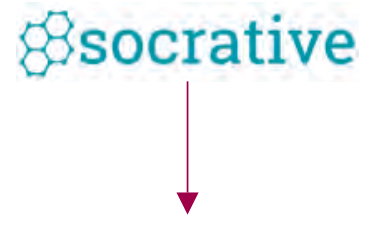

Evaluación Diagnóstica

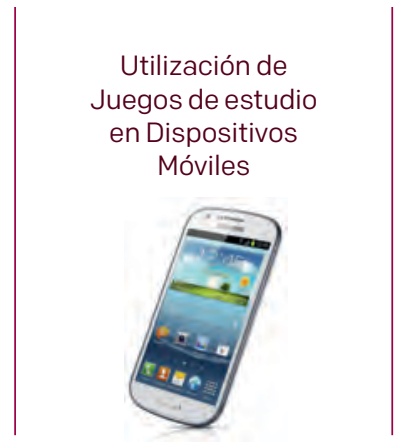

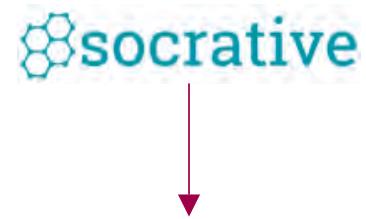

Evaluación Final

Fuente: elaboración propia (2018).

La evaluación diagnóstica permite la realización del censo inicial para determinar la utilización de los dispositivos móviles en la población estudiantil, realizar una evaluación de conocimientos previos con el objetivo de tomar una medición inicial a los estudiantes desde su experiencia o estudios anteriores, y sirve como referencia para determinar su curva de aprendizaje después de la utilización de la metodología.

Una vez utilizada la evaluación diagnóstica, se procede a iniciar la utilización de juegos de estudio que se encuentran disponibles con descarga gratuita en las diferentes tiendas de descargas de los sistemas operativos Android, IOS, Windows Phone.

Se estudiaron diversas aplicaciones que tuvieran las características necesarias para ser utilizadas dentro de la metodología; las aplicaciones se evaluaron teniendo en cuenta los siguientes factores:

1. Plataformas en las que opera: se evaluó de acuerdo con el informe de Gartner (2017), que las plataformas más utilizadas en dispositivos móviles son IOS de Apple, Android y Windows, utilizadas por diferentes marcas.

2. El factor de descargas permite evaluar la cantidad de usuarios que han descargado la aplicación desde un dispositivo móvil.

3. La calificación del usuario es un factor que es tomado directamente de las páginas de descarga AppStore (IOS), PlayStore (Android), Windows Store (Windows). Esta evaluación la realizan únicamente los usuarios que descargaron e instalaron la respectiva 
aplicación, y evalúa conceptos como gráficos, funcionamiento, además de permitir ingresar un comentario. La evaluación se realiza con una métrica de 0-5, y la respectiva tienda realiza un promedio de las evaluaciones.

4. La jugabilidad es una evaluación asignada por los estudiantes, que permite evidenciar que la aplicación tenga diferentes formas de juego, que permita al estudiante una interacción sencilla y que le permita desarrollar un proceso más dinámico para la apropiación del conocimiento, así como tener la posibilidad de una retroalimentación de sus aciertos y errores.

5. Dentro del factor de variedad de preguntas, se evaluó en cada una de las aplicaciones la cantidad de preguntas dentro de la aplicación y si estas preguntas se encuentran dentro de los núcleos temáticos en el estudio de la asignatura Geografía turística.

A continuación se evidencia la respectiva evaluación y análisis de las aplicaciones seleccionadas: Geografía Mundial (Atom Games), Geo Challenge (Wetpalm), Juego de países del mundo (GameSaien.com), Quiz Juego de Geografía (Webelinx), Quiz de Geografía (Quizzes de Peaksel).

En el gráfico 7 se presenta la evaluación del juego desarrollado por Atom Games, llamado "Geografía Mundial", la aplicación mejor evaluada en la interacción con los estudiantes, con una calificación general de 4,7. Dentro de este juego sobresale la variedad y cantidad de las preguntas, diferentes opciones de juego como ubicación geográfica, identificación de ciudades capitales, banderas, monedas, religiones y muchos factores socio demográficos, económicos y culturales; también hay herramientas de idiomas que permiten interactuar y practicar en distintos lenguajes los conceptos geográficos.

Los estudiantes asignan una buena evaluación a sus formas de juego y a sus características. El desarrollo del mismo juego permite a los estudiantes interactuar de diferentes maneras. Al ser una de las aplicaciones mejor valorada por los estudiantes, fue la que mayor impacto tuvo; además, se encuentra en los dos principales sistemas operativos que utilizan los alumnos, Android y IOS.

Por su parte, en el gráfico 8 se encuentra la evaluación realizada a la aplicación/juego "Geo Challenge Geography Quiz", desarrollado por WetPalm para plataformas Android, IOS y Windows. Obtuvo una buena calificación por su jugabilidad, ya que gracias a la calidad de su entorno gráfico, sus variedades, formas y dinámicas del juego, permitían al estudiante interactuar de una manera amena. Esta aplicación tiene una buena calificación global por parte de los usuarios de plataforma, aunque solo incluye dos idiomas. Sus preguntas y la interacción que propone son intuitivas, permitiendo al estudiante desarrollar las actividades de una manera sencilla y ágil. Posee preguntas de ubicación geográfica de lugares del mundo como atractivos turísticos, identificación de elementos como siluetas de mapas y banderas del mundo, por lo que el conocimiento que promueve se ajusta a los requerimientos de aprendizaje de los estudiantes. 
Gráfico 7. Reporte de la evaluación a la aplicación Geografía Mundial, de Atom Games

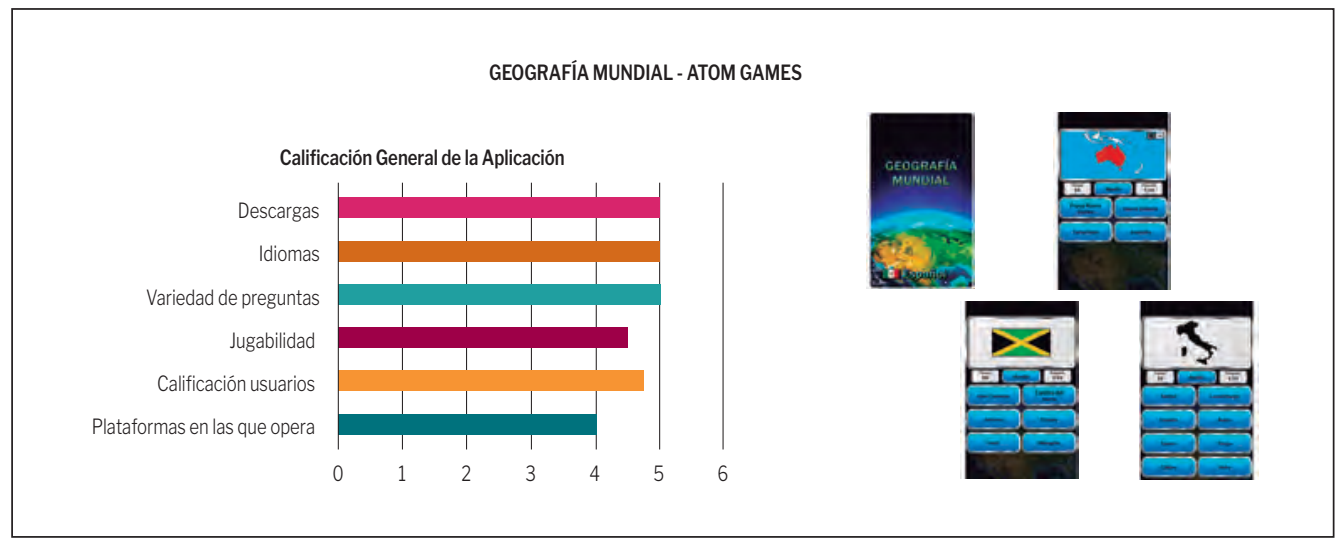

Fuente: elaboración propia, a partir de Atom Games, Geografía Mundial (2018).

Gráfico 8. Reporte de la evaluación de la aplicación Geo Challenge Geography Quiz, de Wetpalm

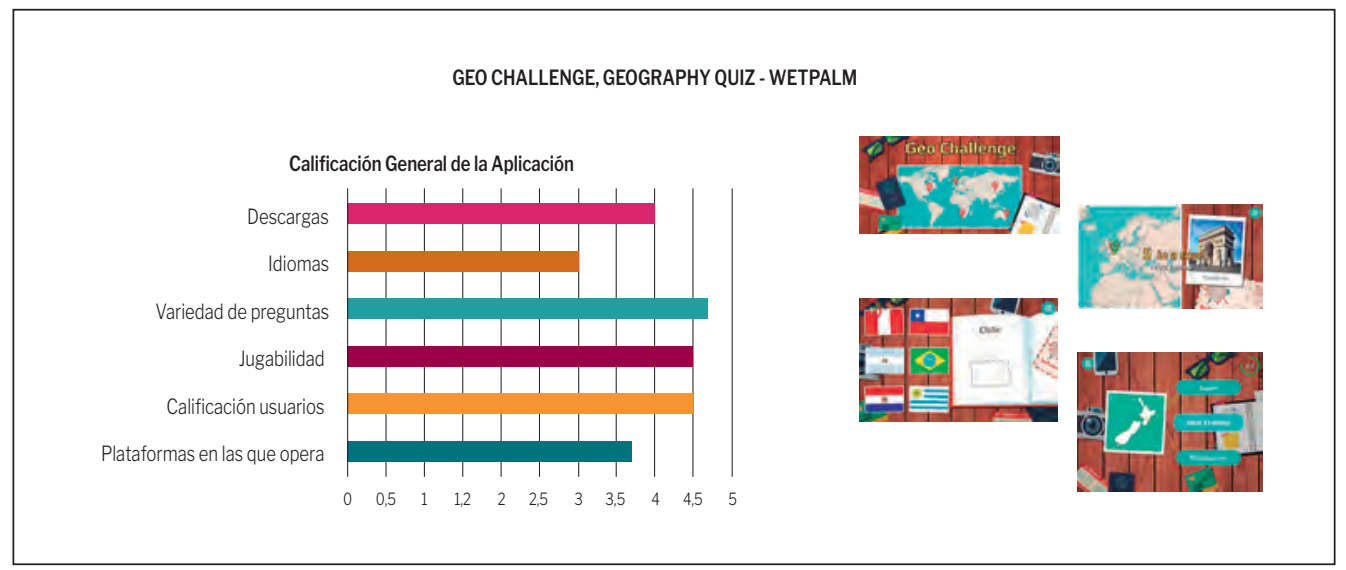

Fuente: elaboración propia, a partir de Wetpalm, Geography Quiz (2018).

En el gráfico 9 se analiza la aplicación/juego “Juego de países del mundo, Juego de Geografía”, desarrollada por la empresa Gamesaien.com. En este caso, la interfaz gráfica no llama tanto la atención de los estudiantes; además, la aplicación tiene dos modalidades de juego que son arrastrar/soltar elementos y preguntas con respuesta de opción múltiple. Tiene un bajo número de descargas, y en este caso el uso de la aplicación por parte de los estudiantes fue inferior al que le dieron a las aplicaciones presentadas previamente. En términos de la información que presenta, posee básicamente preguntas de ubicación geográfica de capitales y ciudades principales del mundo. 
Gráfico 9. Reporte sobre evaluación realizada a Juego de países del mundo, desarrollado por Gamesaien.com

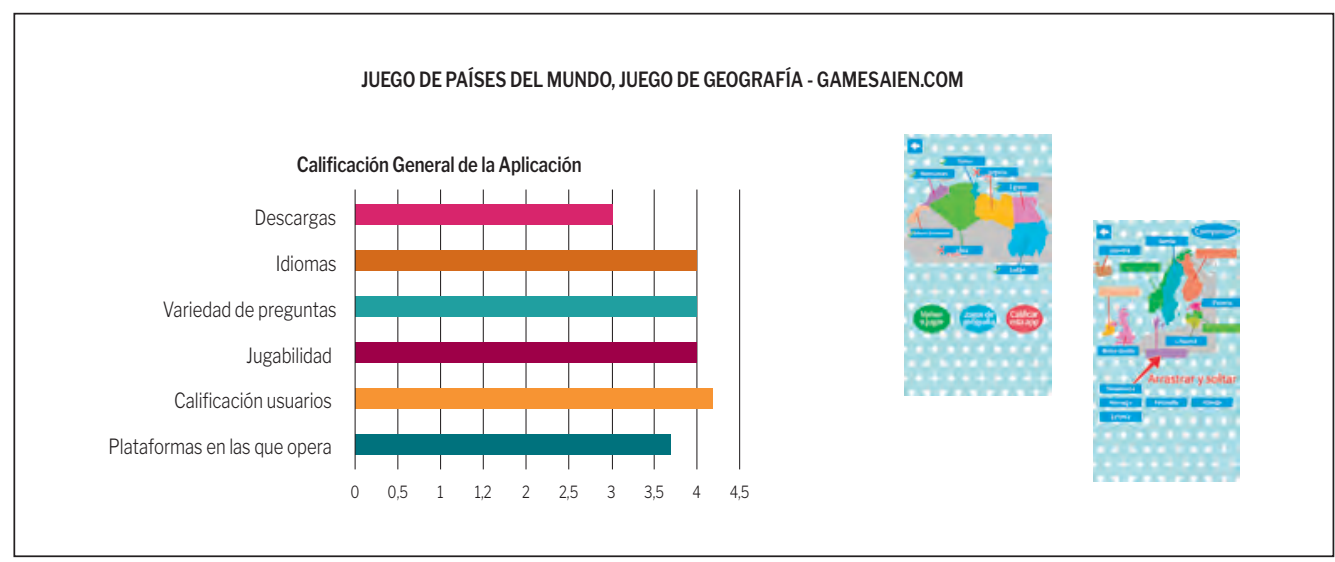

Fuente: elaboración propia, a partir de Gamesaien.com, Juego de Geografía (2018).

En la gráfica 10 se analiza la evaluación realizada a “Quiz, Juego de Geografía”, desarrollada por Webelinx. Es la herramienta que mayor número de descargas tiene en la modalidad de juegos con información geográfica, y por esta razón es la de mayor número de descargas. Se destaca que tiene cuatro modalidades de juego, de 10, 25 y 50 preguntas; sin embargo, su modalidad de preguntas se limita únicamente a respuestas de opción múltiple. Fue popular entre los estudiantes, ya que les permitió compartir sus puntajes por las redes sociales como Facebook, y compartir sus puntajes y premios otorgados por la plataforma, lo que generó un ambiente de competencia sana entre ellos.

Gráfico 10. Evaluación del Quiz- Juego de Geografía, desarrollado por Webelinx

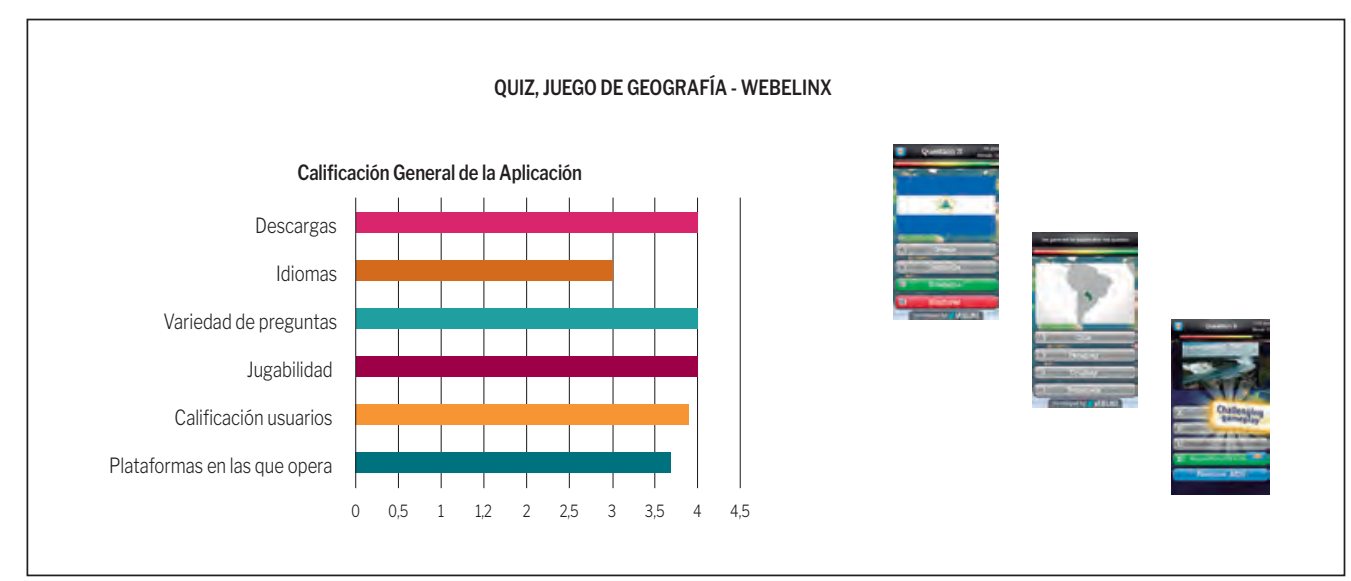

Fuente: elaboración propia, a partir de Webelinx, Juego de Geografía (2018). 
En el gráfico 11 se encuentra el análisis de la aplicación/juego "Quiz de Geografía", desarrollado por Quizzes de Peaksel; esta herramienta tiene buena valoración por su jugabilidad, ya que cuenta con cinco modalidades u opciones de juego como pistas, aaltos de preguntas, corazones de vida, pedir ayuda a un amigo, y además otorga certificados por cada uno de los niveles alcanzados dentro de la dinámica del juego; no tiene variedad de preguntas, ya que se enfoca exclusivamente en interrogantes con respuesta de opción múltiple que se centran en ubicar geográficamente lugares del mundo.

Gráfico 11. Evaluación del juego Quiz de geografía, desarrollado por Quizzes de Peaksel

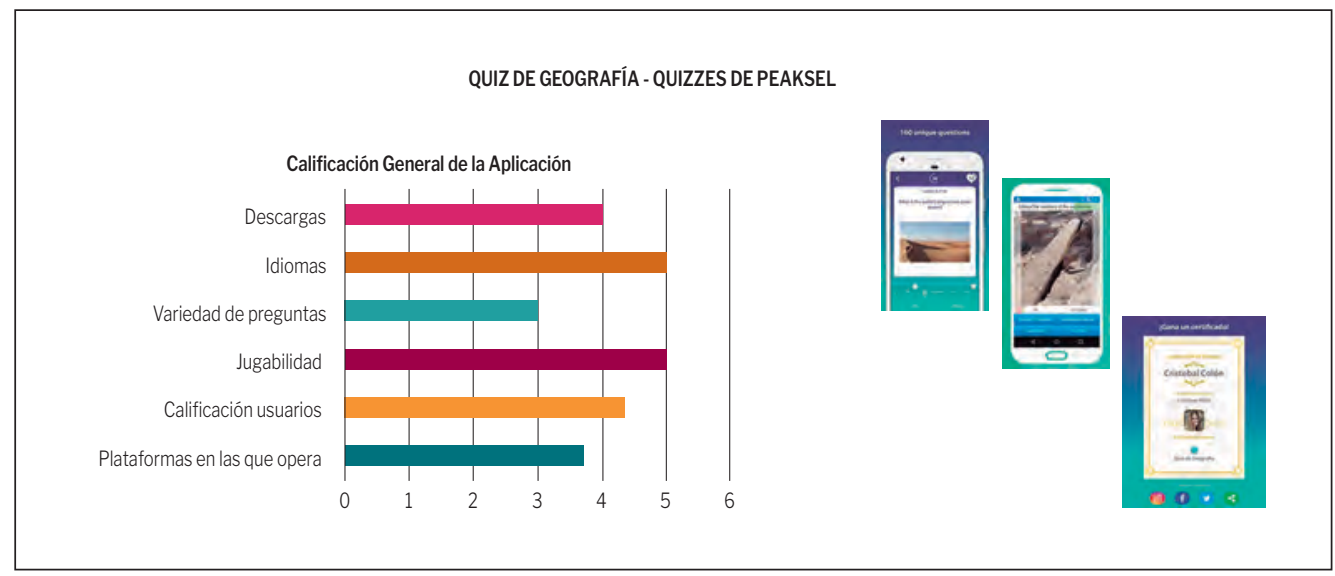

Fuente: elaboración propia, a partir de Quizzes de Peaksel, Juego de Geografía (2018).

\section{Resultados}

La metodología se desarrolló con cuatro diferentes semestres, para un total de 98 estudiantes a quienes, de acuerdo con lo explicado, se les aplicó una evaluación diagnóstica y una evaluación final posterior al uso de los juegos de geografía para aumentar sus competencias anteriormente mencionadas.

La evaluación diagnóstica y la final tiene la misma estructura, sin embargo, las preguntas son aleatorias para determinar un mayor grado de complejidad. La herramienta Socrative tiene cuatro tipos de preguntas que son estilo test, con respuesta de opción múltiple, con una o varias respuestas, preguntas verdadero y falso y respuestas cortas. Los cuestionarios se desarrollaron en cuatro núcleos temáticos:

- Capitales: identificar la capital de un determinado país; nivel de complejidad: bajo.

- Ubicación geográfica: identificar mediante un mapa mudo el país al que corresponde el relieve; nivel de complejidad: alto. 
- Identificación de banderas: identificar mediante una imagen con la bandera el correspondiente país; nivel de complejidad: medio.

- Sitios turísticos: identificar mediante una imagen el correspondiente lugar al que pertenece; nivel de complejidad: medio.

En la ilustración 2 se puede observar un ejemplo de las evaluaciones diagnósticas y finales dentro de la plataforma Socrative; esta actividad la puede desarrollar el estudiante desde un computador o desde el celular.

Ilustración 2. Ejemplo de las evaluaciones diagnósticas realizadas en la plataforma Socrative

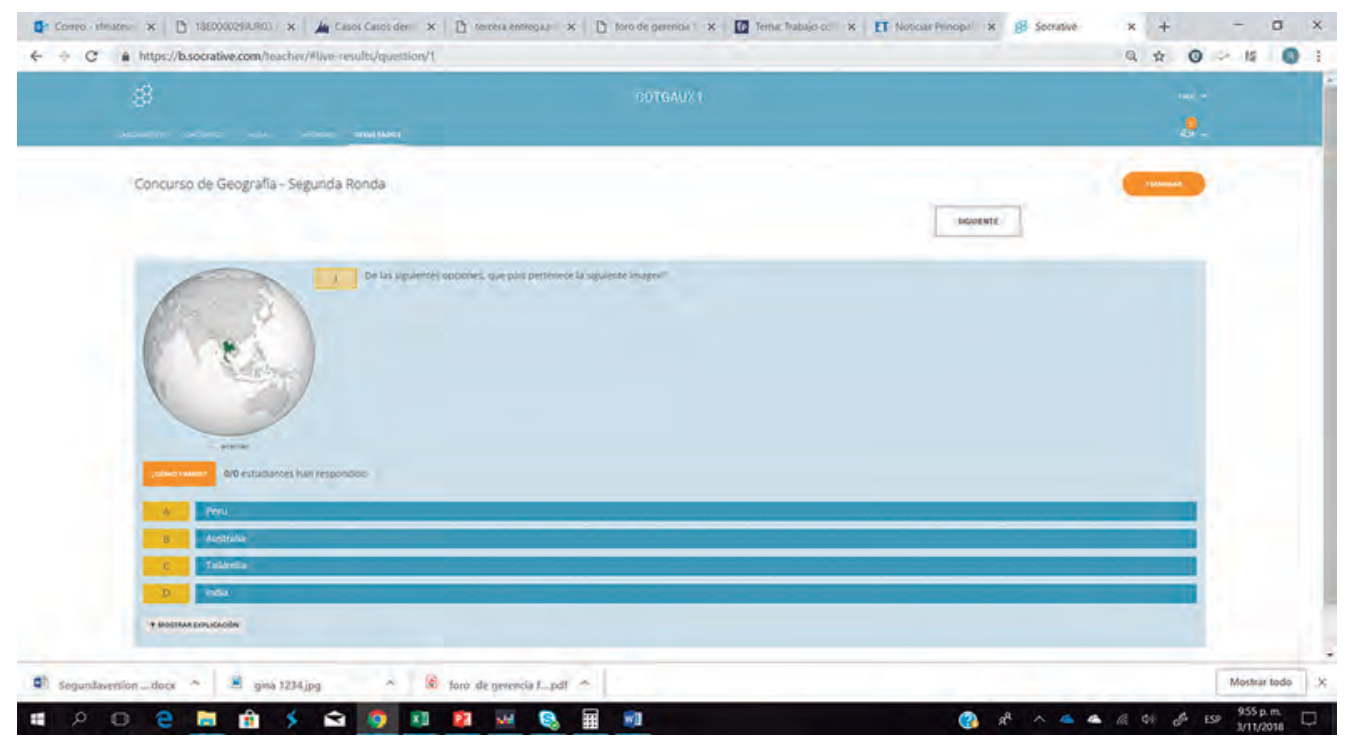

Fuente: elaboración propia, a partir de Socrative (2018).

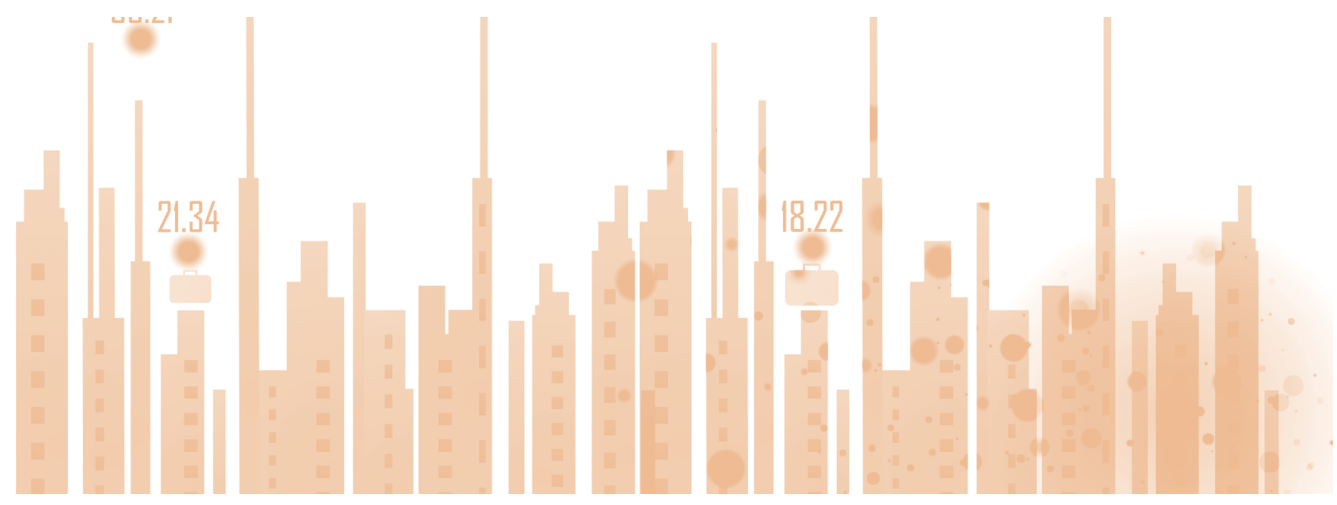


En el gráfico 12 se pueden observar los resultados de los estudiantes en el núcleo temático capitales (cuyo máximo era 100 puntos). Se puede evidenciar que por su baja complejidad, los estudiantes obtuvieron unos buenos resultados en su prueba diagnóstica, y que mejoraron en promedio un 33\% en su medición final.

Gráfico 12. Desempeño de los estudiantes en preguntas núcleo sobre capitales

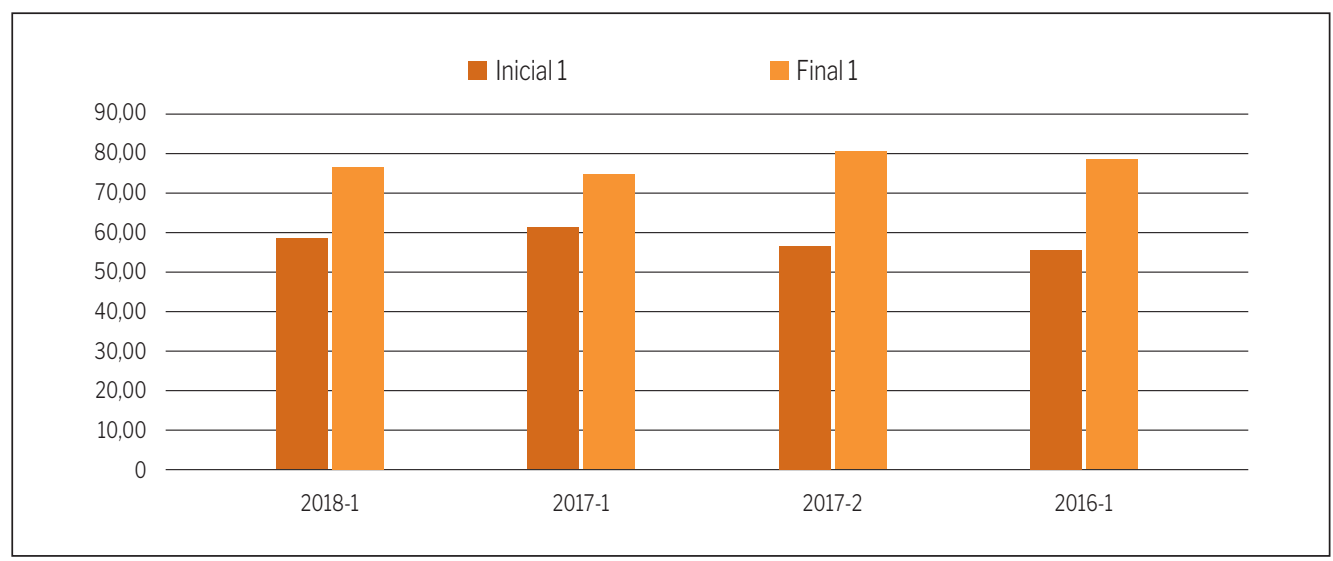

Fuente: elaboración propia (2018).

En el gráfico 13 se puede observar los resultados de los estudiantes en el núcleo temático de ubicación geográfica. Sus resultados mejoraron en un 49\% en promedio, respecto de la medición inicial y la medición final.

Gráfico 13. Desempeño estudiantes en las preguntas núcleo sobre ubicación geográfica

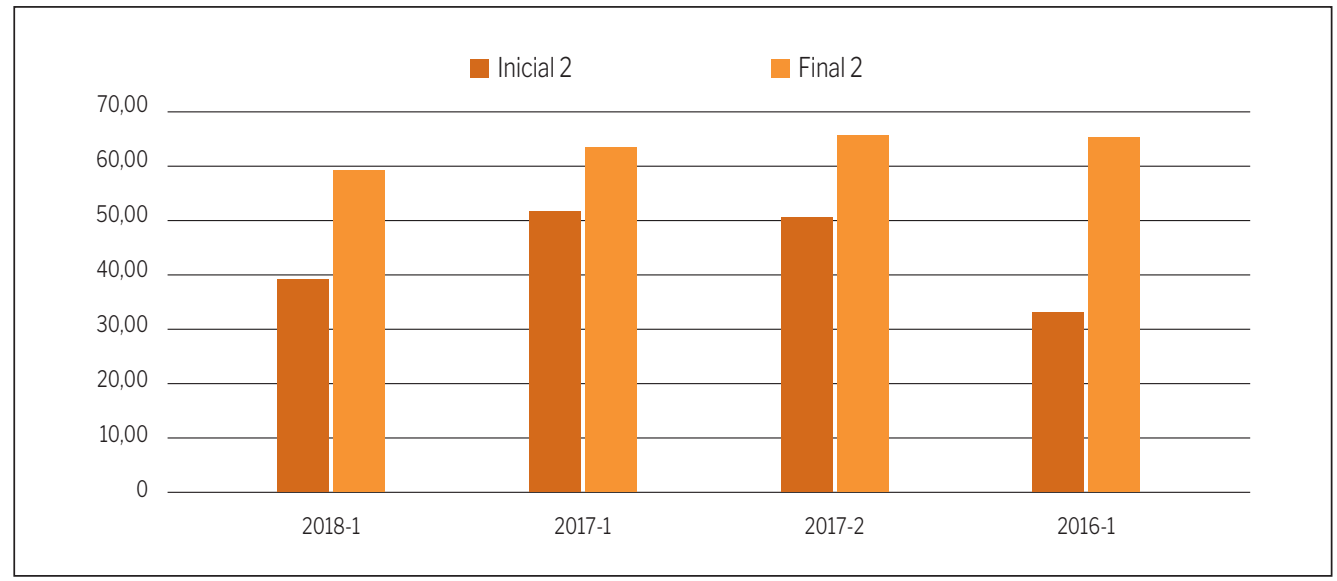

Fuente: elaboración propia (2018). 
En el gráfico 14 se puede observar los resultados de los estudiantes en el núcleo temático de identificación de banderas, a pesar de que uno de los grupos desmejoró en relación con la primera medición; para los estudiantes evaluados entre 2016 y 2017 la mejora entre la evaluación inicial y la final fue de un $24 \%$ en promedio.

Gráfico 14. Desempeño estudiantes en las preguntas núcleo sobre banderas

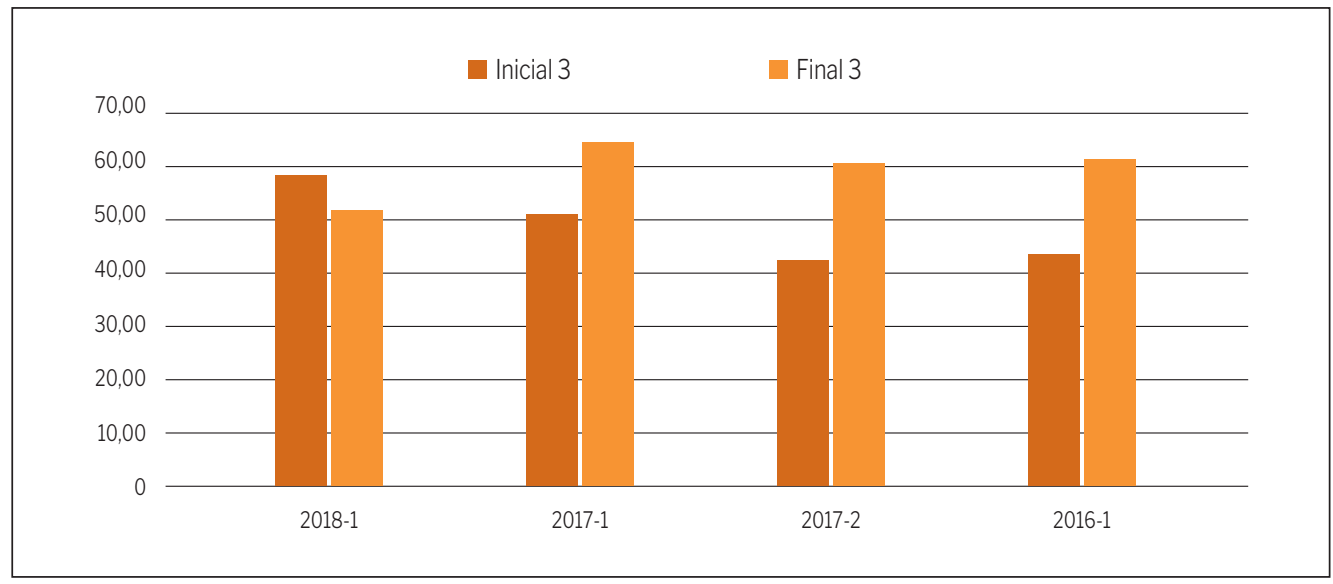

Fuente: elaboración propia (2018).

En el gráfico 15 se puede observar los resultados de los estudiantes en el núcleo temático de sitios turísticos; sus resultados mejoraron en un 18\% promedio, respecto de la medición inicial y la medición final.

Gráfico 15. Desempeño estudiantes en las preguntas núcleo sobre sitios turísticos

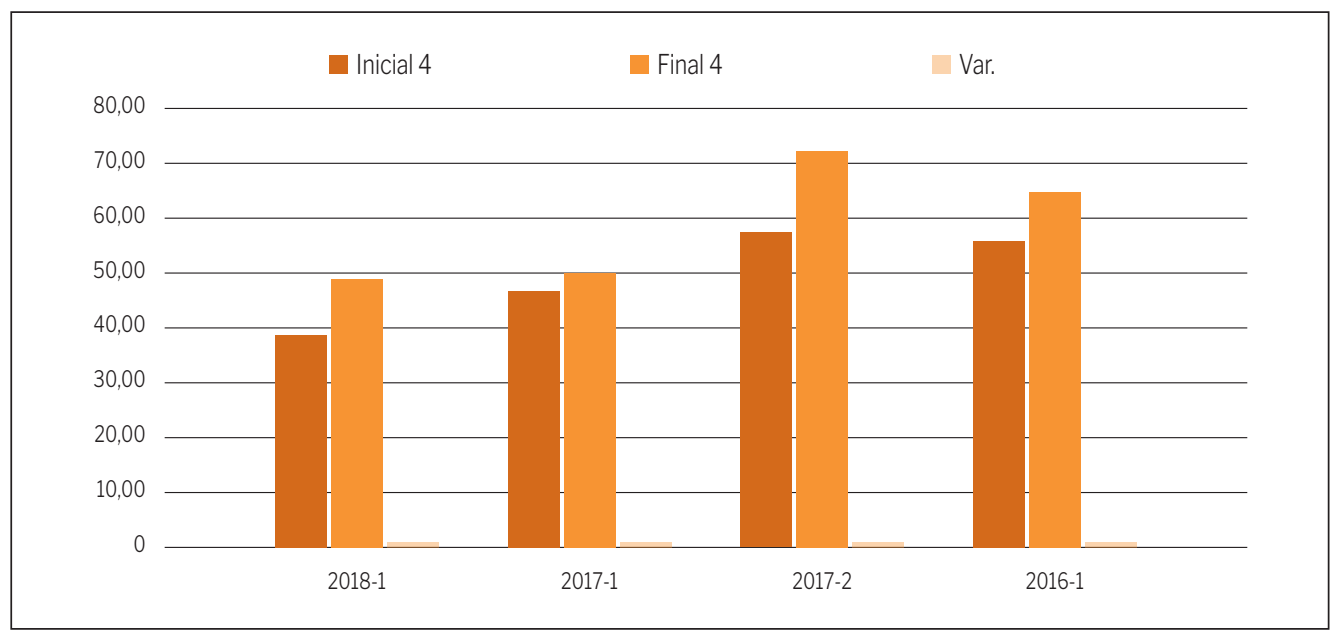

Fuente: elaboración propia (2018). 
En general, el uso de las aplicaciones en el aula de clase se tradujo en un aumento del rendimiento de los estudiantes respecto a la medición inicial. En consecuencia, los dispositivos móviles tienen potencial para convertirse en herramientas que fortalezcan el proceso de enseñanza aprendizaje en el cambio de la gestión de servicios.

\section{Conclusiones}

El aprendizaje mediado por la tecnología se ha venido implementando con éxito en diferentes estrategias y metodologías de participación por parte de los estudiantes y profesores. Para garantizar su éxito se requiere la participación activa y guiada del profesor, para no caer en malas prácticas como producto de los distractores que tienen los mismos dispositivos utilizados.

El ejercicio presentado demuestra que se puede utilizar la tecnología para reforzar el proceso de aprendizaje; si bien el actor principal es el estudiante, los contenidos de los dispositivos móviles pueden servir como herramienta que apoye el proceso de aprendizaje y motive a los alumnos que en la actualidad ven a la tecnología como un aliado.

Si bien en el desarrollo de este trabajo se evaluaron diferentes aplicaciones/juegos que pudieran servir como contenido y como apoyo a las temáticas de la geografía turística, es muy difícil establecer la pertinencia y funcionalidad de todas las preguntas que almacenan en sus bases de datos. Por ello se sugiere mantener una evaluación constante para tratar de evitar que los estudiantes no trabajen con material incorrecto. También se sugiere evaluar el desempeño con cada una de las herramientas para evaluar su potencial educativo.

La utilización de Socrative para evaluar a los estudiantes en la evaluación diagnóstica y la evaluación final permitió identificar que se puede utilizar esta herramienta para establecer dinámicas parecidas a los juegos evaluados y con un material controlado por los profesores, así que para los siguientes periodos se puede pasar a una segunda etapa que implica la creación de bancos de preguntas con los estudiantes y la creación de un juego que le permita al estudiante estudiar en un ambiente más controlado y evaluado que los aplicativos que se consideraron para el estudio.

Los resultados permitieron evidenciar que la metodología aplicada para el estudio de la geografía turística con dispositivos móviles les permitió mejorar sus competencias; con resultados positivos, los estudiantes asimilaron el conocimiento y lo apropiaron de una manera lúdica.

La dinámica de clase y el desarrollo del aprendizaje significativo depende de las diferentes estrategias que los docentes implementen dentro de las aulas: apropiarse de las nuevas tecnologías, no temerles e integrarlas al desarrollo de las clases se traduce en buenos resultados y en un cambio actitudinal por parte de los estudiantes. 


\section{Referencias}

Alonso, J. (2003). Geografía Turística: General y de España. España: Centro de Estudios Ramón Areces.

Boullon, R. (1999). Planificación del Espacio Turístico. Ciudad de México: Trillas.

Brazuelo, F. y Gallego, D. (2012). Mobile learning. Dispositivos móviles como recurso educativo. Bogotá: Eduforma.

Cetra-Centro de tratamiento de adicciones sociales (2005). ¿Existe la adicción al móvil? Valladolid. Recuperado de: http://www.letra.org/spip/ spip.php?article48. Noticias.dot

Atom Games Ent. (2018). Geografía Mundial - Juego (Versión 1.2.109) [Aplicación Móvil]. Descargado de: https://play.google.com/store/apps/details?id=com.age.wgg. appspot\&hl=es

Wetpalm. (2018). Geo Challenge - Geography Quiz (Versión 1.1.2) [Aplicación Móvil]. Descargado de: https://play.google.com/store/apps/details?id=com.wetpalm. GeoChallenge

GameSaien.com. (2018). Juego de Países del Mundo - Juego de Geografía (Versión 1.0.3) [Aplicación Móvil]. Descargado de: https://play.google.com/store/apps/details?i$\mathrm{d}=$ air.com.gamesaien.worldcountriesmapquiz

Webelinx. (2015). Geografía Quiz Juego (Versión 4.3) [Aplicación Móvil]. Descargado de: https://play.google.com/store/apps/details?id=com.quizzes.country.flag.trivia

Quizzes by Peaksel. (2018). Quiz de Geografia (Versión 4.0.0) [Aplicación Móvil]. Descargado de: https://play.google.com/store/apps/details?id=com.geographyquiz. triviagames

Socrative Inc. (2016). Socrative Teacher (Versión 4.4.1) [Aplicación Móvil]. Descargado de: https://play.google.com/store/apps/details?id=com.socrative.teacher 